\title{
CORE ELECTRON DENSITIES OF \\ CORONAL POLAR PLUMES
}

\author{
CASIMIR J. PSUJEK* and RICHARD G. TESKE \\ Dept. of Astronomy, University of Michigan, Ann Arbor, Mich., U.S.A.
}

(Received 20 July, 1972.)

\begin{abstract}
The electron density in the cores of coronal polar plumes that is determined from observations will depend upon the assumed electron density distribution through the plume in a direction normal to its axis. Core electron densities obtained by Saito (1965) and by Newkirk and Harvey (1968) were derived using different assumed electron density profiles, and are not in agreement. We have re-discussed Saito's data using Newkirk and Harvey's electron density profile and find that the disagreement persists. Whether this indicates a true variation in electron density in plume cores cannot now be stated. Errors in the electron densities derived here may arise through errors in measuring the angles $\theta$ and $\alpha$ which enter into the analysis. While plausible variations in $\theta$ produce no appreciable errors in core electron density, plausible variations in $\alpha$ may introduce appreciable errors into the determinations of that quantity.
\end{abstract}

\section{Introduction}

A complete determination of the three-dimensional structure of coronal polar plumes and the electron density along the plumes has been reported on only one occasion. Saito (1965), observing from New Guinea, obtained this information at the February $4 / 5,1962$ eclipse, at phase -0.35 in the solar cycle. He found that, along the plumes, the $\log$ of the core electron density, $\log N_{0}(r)$, was linear with $S=R_{\odot} / r$ between $1.1 \lesssim$ $\lessgtr r \lesssim 1.6 R_{\odot}$, with $\log N_{0}(r) \simeq 8.37$ at $r=1.1 R_{\odot}\left(N_{0}(r)\right.$ is the mean for the plumes discussed).

Incomplete data for other eclipses exist. Saito (1958) determined electron densities along polar plumes (solar cycle phase +0.22 ) under an assumption as to their spatial distribution, finding the average $\log N_{0}\left(r=1.1 R_{\odot}\right) \simeq 7.5$. His results probably did not depend critically upon the assumption made. $\log N_{0}(r)$ was found to be nonlinear with $r$ beyond $r \gtrsim 1.5 R_{\odot}$. Dziubenko (1957) found that $\log N_{0}(r)$ was linear with $\log r$ in polar plumes, but he assumed that they lay in the plane of the sky and took no account of their spatial distribution. Newkirk and Harvey (1968) determined the electron distribution across a 'typical' plume and obtained $\log N_{0}\left(r=1.1 R_{\odot}\right) \simeq 8.0$ near solar minimum, but did not examine the electron density at varying distances from the limb. There is no information yet as to results which might be forthcoming from the March 7, 1970, eclipse, but these data will, in any event, refer to the corona within 15-16 months of solar maximum.

Thus there exists in the literature only one relatively complete analysis of polar plumes, referring to a time almost three years before solar minimum. It is clearly of

* Now at Department of Earth and Space Sciences, SUNY at Stony Brook, New York. 
interest to examine the question as to whether the electron density in polar plumes varies during a cycle.

One way to examine this problem is to compare the results of observations when the methods of analysis are similar. Saito's data and method were used in the work reported here with the exception that for the electron density profile across the plume an analytical electron density distribution due to Newkirk and Harvey (1968) was used. In this way, the observations of Saito and of Newkirk and Harvey may be placed on the same analytical footing and electron densities at two phases in the solar cycle directly intercompared.

\section{Electron Densities}

The core electron densities in the polar plumes were obtained according to the formulae in Saito (1958 and 1965), with one change that we discuss below. The observed brightness $(R)$ of a polar plume at an apparent distance $r^{\prime}$ from the Sun is connected with the core electron density $N_{0}(r)$ at a true solar distance $r$ through the following formulae:

$$
R=\frac{3}{8} \sigma \bar{H}_{\odot} N_{0}(r) w R_{\odot}\left\{A\left(1+\cos ^{2} \alpha\right)+\mathrm{B} \sin ^{2} \alpha\right\} \operatorname{cosec} \omega,
$$

where $R$ is the brightness (expressed in the unit of the Sun's mean surface brightness $\bar{H}_{\odot}=2.62 \times 10^{5} \mathrm{stilb}$ ) of the polarized plus unpolarized components of the plume, $\sigma$ is $0.66 \times 10^{-24} \mathrm{~cm}^{2}$, and $w R_{\odot}$ is the half width of the plume in units of the solar radius in centimeters, and where (Figure 1)

$$
\begin{array}{ll}
\tan \phi^{\prime}=\tan \phi \sin \theta, & \text { (for obtaining } \phi), \\
\tan \left(\phi^{\prime}+\Psi^{\prime}\right)=\tan (\phi+\Psi) \sin \theta, & \text { (for obtaining } \Psi), \\
\cos \omega=\cos \alpha \cos \Psi\left(1+\frac{\tan \Psi}{\tan \phi}\right), & \text { (for obtaining } \omega \text { ), } \\
r \sin \alpha=r^{\prime}, & \text { (for obtaining } r \text { ). }
\end{array}
$$

$A$ and $B$ are as in Saito (1958). In the determination of $A$ and $B$, a limb darkening coefficient equal to 0.75 was used.

Saito (1965) approximated the actual electron distribution across the plume with a uniform distribution across the half-width of the plume, which is defined by Tsubaki et al. (1964) as being a breadth within which the intensity is more than half of the maximum value at the center of it. If the electron density profiles are Gaussian, this should be a valid approximation. Saito (1958) has derived Gaussian profiles of the brightness across two plumes. Newkirk and Harvey (1968) also obtained a Gaussian brightness profile across their plume. From this it can be inferred, not necessarily correctly, that the electron density distribution across a plume is also Gaussian. It appears that this is Saito's reasoning in making his approximation.

Newkirk and Harvey (1968) arrived at an electron density profile across their 'typical' plume of

$$
N(a)=N_{0}\left(1-\frac{a}{3.9 \times 10^{4}}\right)^{1.6} \mathrm{~cm}^{-3}
$$




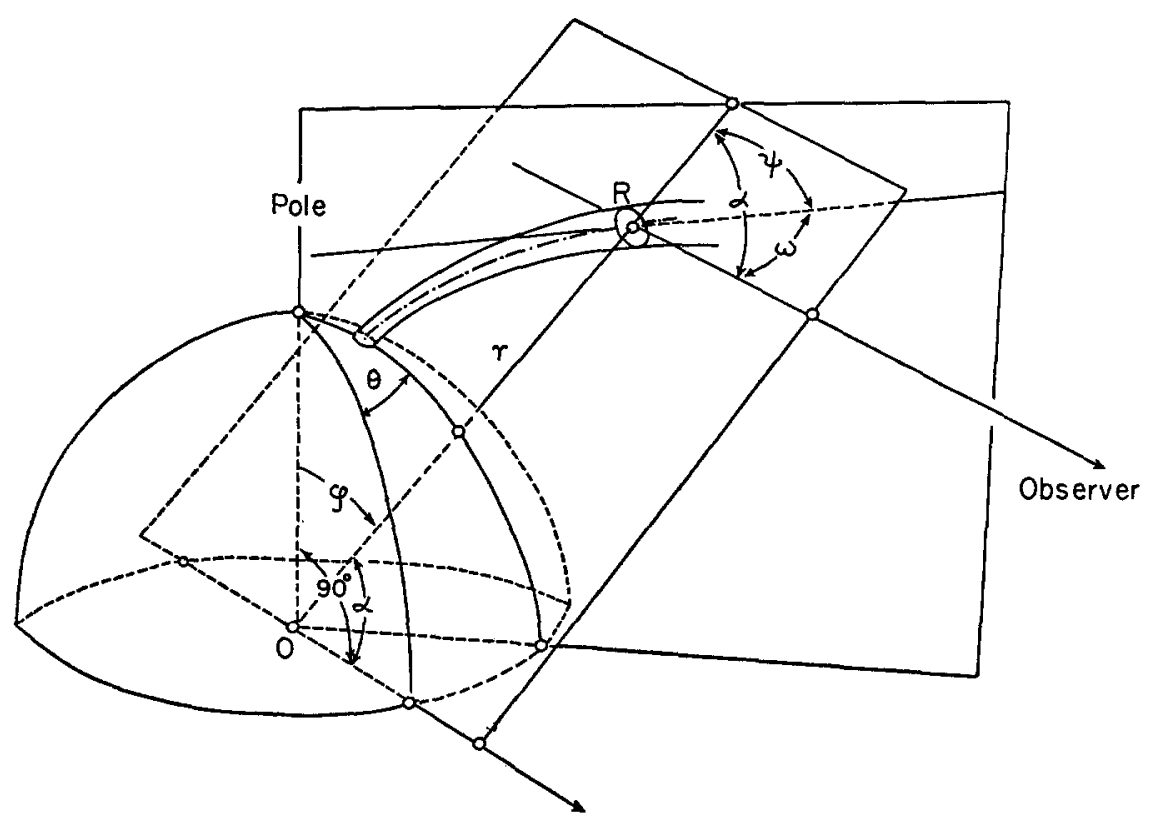

Fig. 1. Diagram for explaining geometric quantities. (From Saito, 1965.)

where $a$ is the distance from the plume axis in kilometers and $N_{0}$ is the core density, at $r=1.1 R_{\odot}$. This electron distribution is not Gaussian, but it provides very nearly a Gaussian brightness distribution.

In our work the assumptions were made that Equation (6) is a 'typical' electron distribution across a polar plume and that this distribution holds for $r$ in the vicinity of $r=1.1 R_{\odot}$, specifically that it is valid at the projected $r^{\prime}=1.1 R_{\odot}$. For $N_{0}(r) w$ in Equation (1), the substitution was made that

$$
N_{0}(r) w=2 \int_{0}^{q} N(a) \mathrm{d} a=2 \int_{0}^{q} N_{0}\left(\frac{q-a}{q}\right)^{1.6} \mathrm{~d} a=(2 / 2.6) N_{0} q,
$$

where $q$ is the radius of the plume at $r=1.1 R_{\odot}$ and is assumed to remain constant for small changes in $r ; q$ is taken to be $3.9 \times 10^{4} \mathrm{~km}$. The result of this substitution is that, if all other parameters in Equation (1) remain the same as in Saito's solutions, his values of $N_{0}$ will be raised somewhat. The new values of $N_{0}$ that are obtained can be seen to be given by

$$
N_{0}(\text { new })=\frac{1.3 w}{q} N_{0} \quad \text { (Saito) }
$$

That all his values of $N_{0}$ will be increased results from the observation that the minimum value of $w$ is $0.046 R_{\odot}$. Specifically, at $r=1.1 R_{\odot}$, the maximum increment to Saito's core electron densities will be $11 \%$ for a plume for which $r=r^{\prime}\left(\alpha=90^{\circ}\right.$ in Equation (5)), since this maximizes $w\left(r^{\prime}\right)$ (see Saito's Equation (5)). 
Saito (1965) lists all the required observational parameters in tabular form except $\alpha, \theta$ and $R$. These can be obtained from figures that the provides. We have re-calculated values of $N_{0}\left(r^{\prime}=1.1 R_{\odot}\right)$ using the values of $\alpha, \theta$ and $R$ given in our Table I. The numbering of the polar plumes is Saito's (1965). In Table II the resulting electron densities are listed.

Most of these values of the plume core electron densities are larger than Saito's by at least $10 \%$, as is required by the use of Newkirk and Harvey's electron density profile. Those of the newly calculated values that vary widely from a small increment of Saito's values are due to difficulties encountered in measuring $\alpha, \theta$ and $R$ on his graphs. The effect of errors in the first two quantities is discussed below. If Saito's core electron densities are all to be raised by the maximum amount, $11 \%$, the resulting mean $\log N_{0}\left(r=1.1 R_{\odot}\right)$ is 8.41 as compared with his average value of 8.37 and with that of Newkirk and Harvey $(\simeq 8.0)$.

Although the scatter in $\log N_{0}(r)$ of the individual plumes is considerable we feel that Saito's (1965) mean value and the 'typical value' of Newkirk and Harvey (1968) are representative of all polar plumes at the times the observations were made. We thus conclude that the differences in core electron density as determined by these authors is not explained by their different assumptions as to the electron density distribution across polar plumes: when Saito's data are re-analyzed using Equation (6), the disagreement is slightly increased. Whether the difference points to a real change of electron density in polar plume cores with phase in the solar cycle cannot yet be stated. Another possible cause of different core electron densities may be short-period (several months or less) fluctuations in the polar magnetic field (Severny, 1971).

It is apparent that the core electron density which is determined from the observations will depend upon the assumed electron density profile across the plumes.

TABLE I

Parameters taken from Saito's (1965) figures $\left(r^{\prime}=1.1 R_{\odot}\right)$

\begin{tabular}{llllllll}
\hline Plume & $\theta$ & $\alpha$ & $R$ & Plume & $\theta$ & $\alpha$ & $R$ \\
\hline 7 & $80^{\circ}$ & $80^{\circ}$ & $10^{-7.3}$ & 15 & 0.5 & $68^{\circ}$ & $10^{-7}$ \\
10 & $49^{\circ} 75$ & $72^{\circ}$ & $10^{-7}$ & 16 & 12.75 & $65^{\circ}$ & $10^{-7}$ \\
12 & $18^{\circ} 75$ & $62^{\circ}$ & $10^{-7.2}$ & 19 & 61.5 & $80^{\circ}$ & $10^{-7}$ \\
13 & 7.75 & $55^{\circ}$ & $10^{-7}$ & 20 & $40^{\circ}$ & $70^{\circ}$ & $10^{-7.1}$ \\
\hline
\end{tabular}

TABLE II

Values for $\log N_{0}\left(r^{\prime}=1.1 R_{\odot}\right)$

\begin{tabular}{llllll}
\hline Polar plume & $\log N_{0}(r)$ & $r$ & Polar plume & $\log N_{0}(r)$ & $r$ \\
\hline 7 & 7.99 & 1.117 & 15 & 8.36 & 1.186 \\
10 & 8.20 & 1.156 & 16 & 8.02 & 1.214 \\
12 & 8.18 & 1.246 & 19 & 8.27 & 1.117 \\
13 & 8.41 & 1.343 & 20 & 8.16 & 1.171 \\
\hline
\end{tabular}


However, as we have shown, the assumptions made by Saito lead to essentially the same core electron density that is found when the Newkirk and Harvey distribution is used.

Saito (1965) found that his values of $\log N_{0}(r)$ varied linearly with $S=R_{\odot} / r$. Since we have found that all his electron density values are increased by the same amount when the Newkirk and Harvey electron distribution is used to describe electron density perpendicular to the plume axes, the linear relationship obtained by Saito is preserved. He has said that the scatter in his values which tends to obscure the linear relation can be explained by difficulties in making small quantity measurements and by systematic errors introduced in determining $\alpha$. Also, as he himself has pointed out, some over-correction may have been made in the conversion of the apparent solar distance $r^{\prime}$ into the true $r$.

\section{Results with Different $\alpha$ and $\theta$}

In determining $\alpha$ and $\theta$ from Saito's graphs, it became apparent that there was room for a certain amount of error in these values and that these errors may not be negligible. An effort was made to ascertain what ranges in density are obtained when $\alpha$ and $\theta$ are varied to a reasonable extent. We felt that, on his published graphs, $\theta$ could be measured with an accuracy corresponding to $\pm 2^{\circ}$ and $\alpha$ with an accuracy of $\pm 10^{\circ}$.

A computer program was written to provide values of the core electron density for various combinations of $\alpha$ and $\theta$. The results were as follows:

(a) The above range in $\theta$ produced no appreciable changes in the value of the core electron density in all the plumes except one. The maximum range in $\log N_{0}(r)$ was 0.03. The one exception was Plume 16. We did not determine why this should be so.

(b) The above range in $\alpha$ produced considerable changes in the value of the core electron density in all the plumes investigated. The maximum and minimum ranges in $\log N_{0}(r)$ were 0.40 and 0.06 respectively. An encouraging note is that although it is easy to make an error of $\pm 10^{\circ}$ when $\alpha>70^{\circ}$, such an error does not introduce as large a change in $\log N_{0}(r)$ as does a similar error when $\alpha<70^{\circ}$ where it is easier to determine $\alpha$ more precisely.

The ranges in the calculated $\log N_{0}(r)$ for two 'typical' plumes are shown in Figures 2(a) and 2(b).
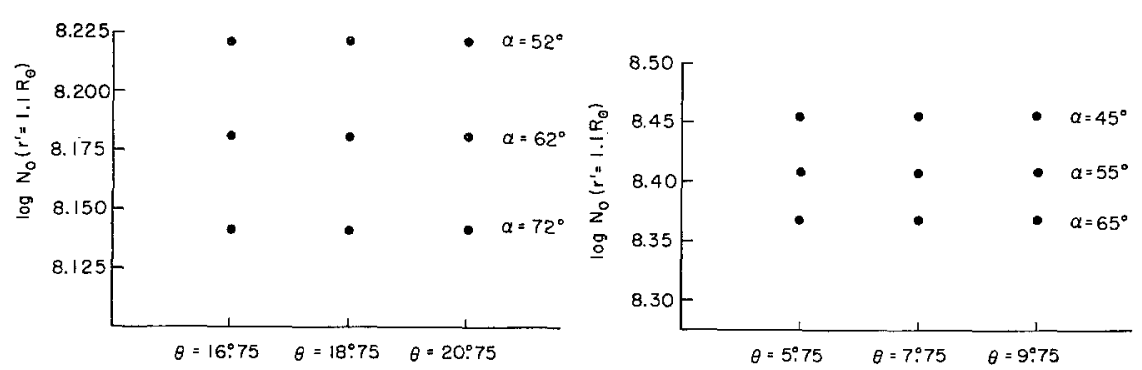

Fig. 2. The diagrams indicate values of $\log N_{0}\left(r^{\prime}=1.1 R_{\odot}\right)$ that are obtained when angles $\alpha$ and $\theta$ are assigned the values shown. (a) Saito's Plume No. 12; (b) Saito's Plume No. 23. 


\section{Conclusions}

(1) The core electron density in coronal polar plumes depends on the form of electron distribution profile across the plume that is assumed.

(2) If we use the Newkirk and Harvey electron distribution profile across a plume, then Saito's values for the core electron density in coronal polar plumes are raised by no more than $11 \%$. The new value is $160 \%$ higher than the one obtained by Newkirk and Harvey.

(3) The logarithm of the core electron density was again found to be linear in $S=R_{\odot} / r$ when the Newkirk and Harvey electron distribution profile is used.

(4) The core electron density is not sensitive to plausible variations in $\theta$, while it is quite sensitive to plausible variations in $\alpha$. The ranges are smaller for large $\alpha$ than for small $\alpha$, if $\alpha$ is varied by an equal amount in both cases.

(5) There is insufficient evidence at the present time to make any statement in regard to how and why there may be differences in the core electron density in coronal polar plumes. However, the difference between values obtained at phase -0.35 (Saito, 1965) and near solar minimum (Newkirk and Harvey, 1968) is not explainable by differences in the analyses.

\section{Acknowledgements}

We would like to thank Drs M. D. Altschuler and K. Saito for their comments on a manuscript of this paper.

\section{References}

Dziubenko, N.: 1957, Soviet Astron. AJ1, 373.

Newkirk, G. and Harvey, J.: 1968, Solar Phys. 3, 321.

Saito, K.: 1958, Publ. Astron. Soc. Japan 10, 49.

Saito, K.: 1965, Publ. Astron. Soc. Japan 17, 1.

Severny, A. B.: 1971, in R. Howard (ed.), 'Solar Magnetic Fields', IAU Symp. 43, 675.

Tsubaki, T., Tominaga, S., Kubota, J., and Kawaguchi, I.: 1964, Publ. Astron. Soc. Japan 16, 13. 